\title{
Dual Parallel Modulation Schemes for Low-Distortion Analog Optical Transmission
}

\author{
STEVEN K. KOROTKY, SENIOR MEMBER, IEEE, AND RENÉ M. DE RIDDER
}

\begin{abstract}
We examine the use of two integrated-optical modulators operated in parallel to attain low-distortion analog optical transmission. This static feedforward approach achieves significantly improved linearity at the expense of small increases in the required optical power and moderate increases in the required drive voltage. A novel variation that uses the unique characteristics of an integrated-optical modulator to correct the dominant quadratic distortion of a directly modulated laser is proposed.
\end{abstract}

A NALOG optical fiber transmission can provide an efficient means of delivering high-frequency signals over long distances. This can be accomplished, for example, by directly modulating the injection current of a semiconductor laser diode and using direct detection. In cases where the signal consists of many frequency multiplexed channels like the composite cable television (CATV) format, the requirements on linearity are particularly stringent.

At present, the second harmonic distortion of directly modulated lasers can be a limitation for CATV applications when the number of frequency multiplexed channels is 40 or more [1]. Thus, feedback and feedforward techniques, as well as alternative modulation schemes, are of interest to attain the necessary optical signal level with low distortion [2]. Here we examine the use of integratedoptical modulators external to the laser source to modulate the optical wave. In particular, we generalize the twopolarization scheme introduced by Johnson and Roussell [3] to the case of a pair of optical transmitters operated in parallel, and analyze the tradeoffs involved. We find that dual parallel modulation schemes provide improved linearity for a given optical signal level at the expense of a small increase of the required optical power and a moderate increase in the required drive voltage. The tolerances on the design parameters are such that practical implementations may require adjustment of the electrical drive signal levels or provisions for trimming of the optical circuit splitters and combiners.

The functional representation of the optical circuit we consider is illustrated in Fig. 1. It consists of two intensity modulators, designated $M_{A}$ and $M_{B}$, that are fed from a single optical source, the outputs of which are combined

Manuscript received November 16, 1989; revised February 14, 1990. S. K. Korotky is with AT\&T Bell Laboratories, Crawfords Corner Road. Holmdel, NJ 07733.

R. M. de Ridder is with the Department of Electrical Engineering, University of Twente, Enschede, The Netherlands.

IEEE Log Number 9036381.

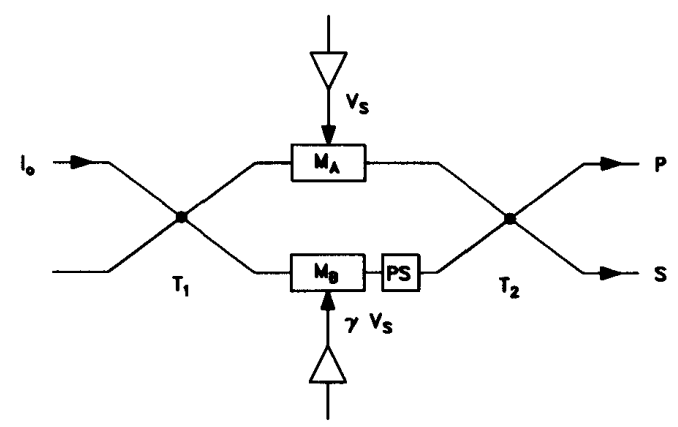

Fig. 1. Schematic of the combination of optical modulators used in parallel to attain low distortion analog modulation. The circuit may include active means (not shown) for setting the dc bias points of the modulators $M_{A}$ and $M_{B}$, the optical taps $T_{1}$ and $T_{2}$, and the phase-shifter $P S$.

to form a single transmitted signal. In practice, the source is a laser and the modulators fundamentally produce amplitude modulation (AM). We assume this is the case, and additionally that the modulators produce AM modulation without a superposed phase modulation, such as can be realized with single-mode waveguide electrooptic modulators [4]. The power splitting and combining ratios of the taps, $T_{1}$ and $T_{2}$, are design parameters. Finally, a means of introducing a static optical phase shift between the two optical amplitudes combined by $T_{2}$ is indicated by the element $P S$.

Using an integrated-optic technology, such as that based on $\mathrm{LiNbO}_{3}$, the above functions may be integrated on a single chip. The modulators are driven by a common signal through individual amplifiers. This provides the flexibility of driving the modulators to different modulation depths. Fabricating the modulators on a single substrate will help ensure that differences in electrical delay times are negligible. Although the modulators are shown spatially separated in the diagram, the modulators may be realized using the two orthogonal polarization states of a single waveguide structure, as originally demonstrated by Johnson and Roussell [3]. Such an approach offers an economy of optical and electrical circuit elements, whereas the use of spatially distinct modulators offers greater flexibility in attaining optimum performance. It should be noted that the static phase shift represented by $P S$ plays fundamentally different roles in these two cases, as will become clear.

The dual parallel modulation schemes considered here 
may be viewed as static, or deterministic, forms of feedforward linearization. One of the modulators is assumed to be driven with an optical input and electrical drive signal that are sufficiently large to exceed the desired signal level. A small amount of the input light is diverted to a second modulator that is driven to a modulation depth larger than the first. Some of this light is then combined with the signal from the first modulator to form the transmitted signal. Because the fraction of the light representing the distortion is larger for the second modulator, only a small amount needs to be added to the output of the first modulator to cancel its dominant distortion component. Also, since the distortion characteristics of the primary and secondary modulator are assumed to be known and dominated by a particular nonlinear coefficient, the distortion need not be assessed dynamically. Thus, the static correction schemes avoid much of the additional highperformance electronic circuitry used in dynamic feedforward compensation [2], [5]. However, it should be noted that some, but not all, of the static correction schemes produce a partial cancellation of the fundamental signal when the dominant distortion term is subtracted out. Thus, there is often an additional optical power penalty associated with the dual modulation approach for eliminating the third-order intermodulation distortion. The fact that one modulator must be driven with a larger signal may also be interpreted as an electrical power penalty. After providing a general analysis of the dual parallel modulation approach, we propose a novel scheme based on an integrated-optical modulator that avoids any cancellation of the fundamental signal.

For purposes of the analysis, we denote the input optical intensity as $I_{o}$ and the corresponding field amplitude as $E_{o}=\sqrt{I}_{o}$. The tap power crossover efficiencies are represented by $\eta_{1}$ and $\eta_{2}$. The fields at the outputs of the modulators are defined as

$$
\begin{aligned}
& E_{A}=\left|E_{A}\right| e^{+i \phi_{A}} \\
& E_{B}=\left|E_{B}\right| e^{+i \phi_{B}}
\end{aligned}
$$

where

$$
\begin{aligned}
& \left|E_{A}\right|=\sqrt{\left(1-\eta_{1}\right)} E_{o} f_{A}(V) \\
& \left|E_{B}\right|=\sqrt{\eta_{1}} E_{o} f_{B}(V) .
\end{aligned}
$$

The functions $f_{A}^{2}(V)$ and $f_{B}^{2}(V)$ describe the intensity versus applied voltage of the modulators $M_{A}$ and $M_{B}$. For a waveguide electrooptic interferometric modulator, $f^{2}(V)=\cos ^{2}(\Delta \beta L / 2)$, where the electrooptically induced phase shift, $\Delta \beta L$, is proportional to the applied voltage.

The field at the primary output port of the device can be written as

$$
E_{P}=E_{A^{\prime}}+E_{B^{\prime}}
$$

where

$$
\begin{aligned}
& E_{A^{\prime}}=\sqrt{\left(1-\eta_{2}\right)}\left|E_{A}\right| e^{+i \phi_{A}} \\
& E_{B^{\prime}}=\sqrt{\eta_{2}}\left|E_{B}\right| e^{+i \phi_{B^{\prime}}}
\end{aligned}
$$

and where the phase shifts introduced by the output combiner and the phase shifter $P S$ have been embedded in $\phi_{B}^{\prime}$.

The electrical current produced in a square-law detector is proportional to the output intensity, which is given by $I=E^{2}=E_{A^{\prime}}^{2}+E_{B^{\prime}}^{2}$

$$
+2\left|E_{A^{\prime}}\right|\left|E_{B^{\prime}}\right| \cos \left(\phi_{A^{\prime}}-\phi_{B^{\prime}}\right) .
$$

If we choose the static phase shift to provide $\phi_{A^{\prime}}-\phi_{B^{\prime}}=$ $\pi / 2$, we have

$$
I=I_{o}\left\{\left(1-\eta_{1}\right)\left(1-\eta_{2}\right) f_{A}^{2}(V)+\eta_{1} \eta_{2} f_{B}^{2}(V)\right\} .
$$

Thus, setting $P S$ to provide a phase shift of $\pi / 2$ creates an effective orthogonality of the fields from modulators $M_{A}$ and $M_{B}$. A similar situation could be obtained using individual light sources for each modulator that are not coherent with each other.

To analyze the distortion characteristics, it is convenient to expand $f_{A}^{2}(V)$ and $f_{B}^{2}(V)$ in powers of the modulation voltage, $V_{m}$, for the bias points of interest. We write

$$
\begin{aligned}
& f_{A}^{2}(V)=a_{0}+a_{1} \nu+a_{2} \nu^{2}+a_{3} \nu^{3}+\cdots \\
& f_{B}^{2}(V)=b_{0}+b_{1} \gamma \nu+b_{2} \gamma^{2} \nu^{2}+b_{3} \gamma^{3} \nu^{3}+\cdots
\end{aligned}
$$

where the $a_{k}$ and $b_{k}$ are the Taylor expansion coefficients for the chosen bias points, $\nu=V_{m} / V_{S}$ is the modulation voltage normalized to the switching voltage $V_{s}$, and $\gamma$ accounts for the possibility of a different drive signal level or switching voltage for the two modulators. With these definitions we can write

$$
I=I_{o}\left\{c_{o}+c_{1} \nu+c_{2} \nu^{2}+c_{3} \nu^{3}+\cdots\right\}
$$

where

$$
c_{k}=\left(1-\eta_{1}\right)\left(1-\eta_{2}\right) a_{k}+\eta_{1} \eta_{2} \gamma^{k} b_{k} .
$$

The optical signal of interest is $I_{S} \simeq I_{o} c_{1} \nu$. We define the normalized drive index, $m_{i}$, as $m_{i}=2\left\|V_{m}\right\| / V_{S}$ and the optical modulation depth (OMD) $m_{o}$ as

$$
m_{o}=\frac{I_{\max }-I_{\min }}{I_{\max }+I_{\min }}=\frac{c_{1}}{c_{0}} \frac{\left\|V_{m}\right\|}{V_{S}} .
$$

The second-order two-tone products $(2 I M)$ have a power relative to the carrier frequency of

$$
\frac{P_{2 I M}}{P_{C}}=\frac{c_{2}^{2} c_{0}^{2}}{c_{1}^{4}} m_{o}^{2} .
$$

The third-order two-tone products $(3 I M)$ are at a level below the carrier of [6]

$$
\frac{P_{3 I M}}{P_{C}}=\frac{9}{16} \frac{c_{3}^{2} c_{0}^{4}}{c_{1}^{6}} m_{o}^{4} .
$$

To compare the relative merit of methods to improve linearity, it is useful to note the theoretical performance of a baseline system using external modulation and the approximate requirements for a 40-channel CATV optical link. We consider a system consisting of a CW laser pro- 
viding $100 \mathrm{~mW}$ output power and relative intensity noise (RIN) of $-160 \mathrm{~dB} / \mathrm{Hz}$, lens and isolator with loss of 3.5 $\mathrm{dB}$, interferometric modulator having excess loss of $3 \mathrm{~dB}$, link loss of $5 \mathrm{~dB}$, and receiver with noise of 15 $\mathrm{pA} / \sqrt{\mathrm{Hz}}$. If the modulator is biased for negligible quadratic distortion, the Taylor coefficients are: $c_{0}=0.5, c_{1}$ $=1.57, c_{2}=0$, and $c_{3}=2.58$ [6]. For an OMD of $3 \%$, a carrier-to-noise ratio of $\sim 55 \mathrm{~dB}$ is obtained. For the same modulator drive conditions, the distortion is characterized by two-tone third-order intermodulation products at levels of $-79 \mathrm{dBc}$. The requirement on the twotone $3 I M$ products to ensure satisfactory performance of a 40 -channel AM CATV system is $\sim-90 \mathrm{dBc}$. Thus, if using a distortion compensation technique, it must reduce the $3 I M$ products by $11 \mathrm{~dB}$ for the system and conditions just described. We note, however, in the shot noise limit, that this reduction could alternatively be attained by reducing the modulation depth to $\sim 1.6 \%$ and increasing the transmitter power by $2.7 \mathrm{~dB}$. Thus, there is motivation to consider using external modulation with very high power optical sources having low RIN. At the same time, the advantage of reducing the distortion level without significant optical power penalty is clear.

We will examine the application of the dual modulator formalism to several cases. First, we consider the class of circuits in which $M_{A}$ and $M_{B}$ are interferometric modulators. A set of static phase bias voltages is used to obtain $a_{1}=-b_{1}, a_{3}=-b_{3}$, which may be accomplished by biasing $M_{A}$ at $\Delta \beta L=+(\pi / 2)$ and $M_{B}$ at $\Delta \beta L=-(\pi / 2)$ on the interferometers' switching curves. (In the dual polarization case, $P S$ is used to attain this relative inversion.) At these points, we also have $a_{0}=b_{0}=1 / 2$ and $a_{\text {even }}=b_{\text {even }}=0$. To eliminate the cubic term in (8), we require that $c_{3}=0$, or

$$
\left(1-\eta_{1}\right)\left(1-\eta_{2}\right)=\eta_{1} \eta_{2} \gamma^{3} \text {. }
$$

With this condition satisfied, the residual distortion level falls to a substantially lower value predominately determined by the fifth-order term of (8). The ratio of the linear optical signal strength from the primary output port compared to that of a single conventional interferometric modulator identical to $M_{A}$ is, using (9),

$$
R=\left(1-\eta_{1}\right)\left(1-\eta_{2}\right)-\eta_{1} \eta_{2} \gamma
$$

Various configurations of the dual interferometers are distinguished by the boundary conditions placed on the output tap $T_{2}$. It can be noted, for example, that for any value of $\gamma$, a unique set of values for $\eta_{1}$ and $\eta_{2}$ maximizes the value of $R$. The relationship between the increase in required drive voltage and reduced fundamental signal may be expressed as an optical versus electrical drive power tradeoff. We define the electrical power penalty as

$$
P_{E}=20 \log _{10} \gamma
$$

and the optical power penalty as

$$
P_{O}=-10 \log _{10} R \text {. }
$$

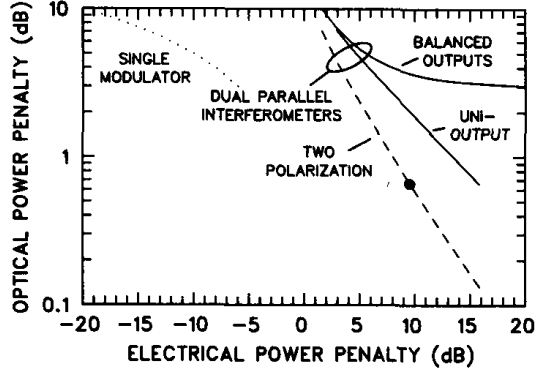

Fig. 2. Relationships between effective electrical and optical power penalties for the dual parallel modulation schemes.

The relationship between $P_{O}$ and $P_{E}$ for the values of $\eta_{1}$ and $\eta_{2}$ satisfying (13) and optimizing $R(14)$ is plotted in Fig. 2 and is identified by the label "uni-output." For comparison, the curve labeled "single modulator" represents the tradeoff relationship when the drive signal level to a single external modulator is reduced from $3 \%$ so as to attain a $3 I M$ of $-90 \mathrm{dBc}$ or lower. The minimum optical penalty is $-3 \mathrm{~dB}$.

A second configuration of the dual interferometer structure corresponds to the case of equal output levels from the two ports of $T_{2}$, i.e., $\eta_{2}=1 / 2$, and is labeled "balanced outputs." This configuration results in a minimum effective insertion loss of $3 \mathrm{~dB}$, although two transmitter outputs are provided. At a value of the optical power penalty of $4 \mathrm{~dB}$, the required drive voltage increase is approximately $\gamma=2.6 \times$. Thus, with this approach, a system that far exceeds the distortion requirement, provides two transmitter outputs, and has sufficient margin for transmission can be realized. Flexibility in the adjustment of $\gamma$ provides a means of optimizing the performance in the presence of small fabrication variations in the values of either $\eta_{1}$ and $\eta_{2}$. The worst-case distortion and change in excess loss introduced by such errors may be assessed using (13) and (14). If the value of $\eta_{2}$ is correct, then no degradation in distortion is incurred and the optical versus electrical tradeoff is as indicated in Fig. 2. If the value of $\eta_{1}$ is correct and $\gamma=2.6$ is not changed, then the twotone distortion specification of $3 I M=-90 \mathrm{dBc}$ at OMD $=3 \%$ is met for $\eta_{2}$ in the range of $0.49<\eta_{2}<0.51$. This is a moderately stringent requirement to realize with a passive design. Given the ability to adjust $\gamma$, no restriction is placed on $\eta_{2}$ to meet the specification for the primary port; however, the distortion performance of the secondary output port is sacrificed. Note: if the secondary port is not to be used, then the "uni-port" design will provide a lower optical insertion loss.

The device reported in [3], which makes use of the two orthogonal polarization modes of the optical waveguides, is a special case of the balanced configuration just described. The signals on both polarizations may be carried to a single detector to realize a larger received signal level, as indicated by the curve labeled "two polarization." The datum corresponding to the single-drive electrode $\mathrm{Ti}: \mathrm{LiNbO}_{3}$ device of Johnson and Roussell is indicated 
by the filled circle on that curve. That device operates with a value of $\gamma=3$, determined by the ratio of the electrooptic efficiencies for the two polarizations, and with an excess optical loss of $\sim 0.7 \mathrm{~dB}$. In addition to the higher power level in the single output, a second potential advantage of the two-polarization technique worth noting is that since a single electrode is used to affect the modulation, only a single electrical drive amplifier is required. These improvements, however, are attained at the expense of restrictions on the values of $\gamma$ that are attainable, and the increased complexity needed to ensure the correct polarization ratio is launched. As the value of $\gamma$ is determined by the electrode and waveguide designs and the crystal properties, only $\eta_{1}$ may be adjusted to compensate for fabrication variations in $\gamma$ or differential TE / TM optical loss. The range of $\eta_{1}$ that provides twotone $3 I M$ distortion products below $-90 \mathrm{dBc}$ for $\mathrm{OMD}=$ $3 \%$ when $\gamma$ is fixed at $\gamma=3$ is $0.034<\eta_{1}<0.037$. Thus, a practical implementation of the two-polarization technique may require active adjustment of the polarization splitting ratio during the fiber-pigtailing process, an active polarization control element at the input, or an adjustable polarization splitter at the output. Note: the acceptable range of $\eta_{1}$ becomes larger for smaller values of $\gamma$.

As a final example, we extend the parallel modulation approach to include the case of a directly modulated laser in combination with a second laser that is operated $\mathrm{CW}$ and externally modulated. A means of combining portions of the outputs of each is provided by a coupler, which we again designate as $T_{2}$. The fiber-coupled optical power output of the CW laser is $I_{o}$ and is assumed to be twice the time-averaged optical power output of the directly modulated laser. We also suppose that the modulation depth of the directly modulated laser is $4 \%$ and that it provides the desired fundamental signal level with negligible $3 I M$ distortion. The $2 I M$ distortion level of this laser is taken to be $-60 \mathrm{dBc}$. The insertion loss of the external modulator is assumed to be $3 \mathrm{~dB}$. However, in contrast to the previous examples, the external modulator $M_{B}$ is biased to one of the operating points where the magnitude of $b_{2}$ is maximum, i.e., where $\Delta \beta L$ is an integer multiple of $\pi$. The choice of the operating point will depend on whether a positive or negative value of $b_{2}$ is necessary to cancel the laser distortion. If we express the response of the laser in the form of (7) and (11) with coefficients $d_{k}$ and assume the lasers are not coherent with each other, then the requirement to eliminate the $2 I M$ distortion becomes

$$
m_{i}=\frac{d_{0}}{d_{1}} \sqrt{\frac{2 d_{2}\left(1-\eta_{2}\right)}{b_{2} \eta_{2}}} m_{o}
$$

where $m_{i}$ is the modulation index of the external modulator, and $m_{o}$ is the optical modulation depth of the laser. The reduction factor for the fundamental signal from the primary output is $R=\left(1-\eta_{2}\right)$. For the operating and distortion values of the present example, the laser coefficients are approximated by $d_{0}=1 / 2, d_{1}=1,\left|d_{2}\right|=$ 0.05 , and $m_{o}=0.04$. The maximum magnitude of $b_{2}$ is $\pi^{2} / 4[6]$ and we assume $\eta_{2}=0.2$. In this case, a value of $m \leqslant 0.01$ will provide the necessary cancellation of the $2 I M$ at the primary output with less than a $1 \mathrm{~dB}$ optical power penalty. Also, since the secondary transmitter does not introduce any cancellation of the fundamental signal, the $20 \%$ reduction in the optical signal level in the output coupler can be eliminated by increasing the drive current by the same factor, provided the laser rating is not exceeded. Another option is to arrange for the optical polarization from the compensating modulator to be orthogonal to that of the primary modulation and to use a polarization combiner for $T_{2}$. Finally, we note that the optical power level from the $\mathrm{CW}$ laser and the external modulator modulation index may be adjusted to optimize the performance for any fixed value of $\eta_{2}$. This may be automated using a pilot signal and slow feedback loop. Thus, this approach may represent a viable option to attaining low distortion analog modulation.

In summary, we have analyzed the general case of the use of two optical modulators connected in parallel to attain low distortion analog optical transmission. The approach achieves significantly improved linearity at the expense of small increases in the required optical power and moderate increases in the required drive voltage. However, relatively stringent requirements are placed on control of the design parameters to attain the $3 I M$ distortion performance necessary for high-capacity CATV systems. Practical implementations may require a means of trimming the electrical drive signal levels or coupler crossover efficiencies to account for variations in device fabrication. A promising variation on the approach that uses a combination of both direct and external modulation was proposed.

\section{ACKNOWLEDGMENT}

It is a pleasure to acknowledge F. Heismann for fruitful discussions on the subject and constructive comments on this manuscript.

\section{REFERENCES}

[1] A. A. M. Saleh, "Fundamental limit on number of channels in subcarrier-multiplexed lightwave CATV system, " Electron. Lett., vol. 25, p. $928,1989$.

[2] R. E. Patterson, J. Straus, G. Blenman, and T. Witkowicz, "Linearization of multichannel analog optical transmitters by quasi-feedforward compensation technique," IEEE Trans. Commun., vol. COM-27, p. $582,1979$.

[3] L. M. Johnson and H. V. Roussell, "Reduction of intermodulation distortion in interferometric optical modulators,"' Opt. Lett., vol. 13 , p. 928,1988

[4] F. Koyama and K. Iga, "Frequency chirping in external modulators," J. Lightwave Technol., vol. 6, p. 87, 1988.

[5] R. M. de Ridder and S. K. Korotky, "Feedforward compensation of integrated-optic modulator distortion," in Tech. Dig. Conf. Opt. Fiber Commun, San Francisco, CA, 1990, paper WH5.

[6] T. R. Halemane and S. K. Korotky, "Distortion characteristics of optical directional coupler modulators," IEEE Trans. Microwave Theory Tech., vol. 38, pp. 669-673, 1990 


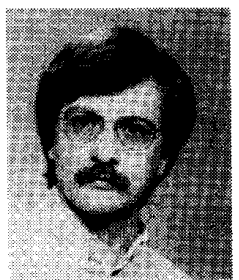

Steven K. Korotky (M'87-SM'87) was born in Paterson, NJ, on February 13, 1953. He received the B.A. degree in physics from Rutgers University, New Brunswick, NJ, in 1975, and the M.S. and Ph.D. degrees in physics from Yale University, New Haven, CT, in 1976 and 1980.

He joined AT\&T Bell Laboratories in 1980 and is a Distinguished Member of Technical Staff in the Photonic Circuits Research Department, Holmdel, NJ. His research there is in the area of integrated-optic devices and experimental lightwave systems with emphasis on high-speed optical modulation and switching.

Dr. Korotky is a member of the American Physical Society.

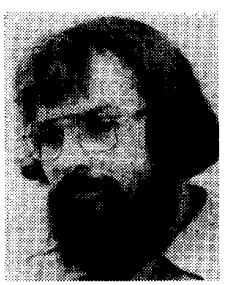

René M. de Ridder was born in Amsterdam, The Netherlands, on April 26, 1950. He studied electrical engineering at the University of Twente, Enschede, The Netherlands, where he received the Masters degree in 1978 and the Ph.D. degree in 1988.

Since 1981 he has been employed as a Lecturer and Research Scientist at the University of Twente. His current research interest is in integrated-optic devices based on nonlinear effects in polymers. 